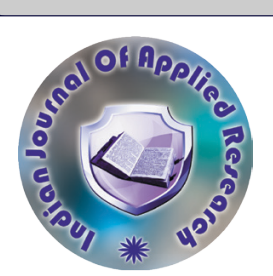

General Surgery

\title{
EFFECT OF ACUTE APPENDICITIS AND ITS COMPLICATIONS ON THE PARAMETERS OF LIVER FUNCTION TESTS
}

\section{Dr. Shouryabrata Choudhury* \\ Dr. Neelanjana Paul \\ Dr. Bhaskar Sharma}

Assistant Professor, Dept. of General Surgery, Silchar Medical College and Hospital Silchar. *Corresponding Author

PGT, Dept of General Surgery, New PG Hostel, SMCH, Silchar-788014.

ABSTRACT BACKGROUND AND OBJECTIVES: Acute appendicitis is one of the most common surgical conditions encountered by the surgeons worldwide. In acute appendicitis, liver function test is one of the biochemical tests used to predict the severity of disease process. Blood from the abdominal organs reaches the liver via portal vein which not only carries the nutrients but also bacteria and toxins absorbed from the gut. When the load is more than the functional capacity of the liver, it leads to parenchymal damage to the liver, which is reflected as deranged parameters of liver function tests. The present study has been designed to evaluate the effect of acute appendicitis and its complications on liver function tests.

MATERIAL AND METHOD: This observational prospective study was conducted on 55 cases of acute appendicitis and its complications. Patients of all ages and of either sex presenting with acute appendicitis (ALVARADO score $>9$ and confirmed by USG) were included in the study and were subjected to Liver function tests (LFT) and histopathology examination report were collected after appendicectomy.

RESULTS: On histopathological and intraoperative examination, $5.45 \%$ had normal appendix, $54.54 \%$ had inflamed appendix, $40 \%$ had gangrenous or perforated appendix. Liver function tests were evaluated in these subjects. We found that in subjects with inflamed appendix, $16.6 \%, 10 \%$ and $6.6 \%$ had elevated levels of total bilirubin, AST, ALT respectively and none had elevated ALP. Similarly, in subjects with perforated/gangrenous appendix, $72.7 \%, 54.5 \%, 18.2 \%$ and $9.1 \%$ had elevated total bilirubin, AST, ALT and ALP levels respectively. The LFT parameters are deranged in pathological appendix and more specifically the total bilirubin level is high for gangrenous and perforated appendix. CONCLUSION: LFT parameters are deranged in complicated appendicitis and more specifically the total bilirubin level.

KEYWORDS : Acute appendicitis, Liver function tests, bilirubin level

\section{INTRODUCTION}

Acute appendicitis is one of the most common surgical conditions encountered by the surgeons worldwide. Early diagnosis and intervention play a key role in decreasing mortality and morbidity associated with the condition.

The classical clinical features of appendicitis may not always be present. Various scoring systems, laboratory tests, and imaging studies are employed for an accurate and early diagnosis of acute appendicitis. But these are not specific for the diagnosis severity of acute appendicitis and complications.

Liver function tests (LFT) is one of the biochemical tests used to predict the severity of disease process in acute appendicitis. Recent research literatures have shown that hyperbilirubinemia is a diagnostic tool for complications of acute appendicitis. Blood from the abdominal organs reaches the liver via the portal vein which not only carries the nutrients but also bacteria and toxins absorbed from the gut. In appendicitis, there is mucosal ulceration which facilitates the invasion of the bacteria into muscularis propria, that leads to acute suppurative appendicitis. Transmigration of bacteria occurs through the appendicular wall and reaches the liver through portal vein. When the load is more than the functional capacity of the liver, it leads to parenchymal damage in the liver, which is reflected as deranged parameters of liver function test. There is also local release of cytokines like TNF, IL-6 in the liver parenchyma in response to the bacterial load leading to depressed excretory function of liver and may cause increase in serum bilirubin level without rise in liver enzymes. ${ }^{1,2,3}$ The present study has been designed to evaluate the effect of acute appendicitis and its complications on parameters of liver function test.

\section{AIMS AND OBJECTIVES}

The aim of the present study is to study the effect of acute appendicitis on liver function tests and also evaluate the sensitivity, specificity, positive predictive value (PPV) and negative predictive value (NPV) of the different LFT parameters in complications of acute appendicitis.

\section{MATERIALAND METHOD}

The present study is an observational prospective case study and was conducted on 55 patients admitted in the Department of General
Surgery, Silchar Medical College and Hospital, Assam, over a period of 6 months, from March 2020 to August 2020. Patients of all ages and of either sex presenting with features of acute appendicitis and its complications (ALVARADO score $>9$ and confirmed by USG) were included in the study. Patients with acute appendicitis with history of hepatotoxic drug intake, past history of jaundice and chronic liver disease and those managed conservatively were excluded from the study.

After taking informed consent, patients were subjected to thorough clinical evaluation, routine blood tests, LFT and USG whole abdomen. Post-appendicectomy samples were subjected to histopathological examination and reports were then collected. Data collection was compiled in a systemic way in preformed proforma and analyzed using SPSS ver. 20.

\section{RESULTS AND OBSERVATIONS}

A total of 55 cases were enrolled in the study who had been operated for Acute appendicitis and its complications. Out of 55 subjects, 30 were males and 25 were females. The age group commonly involved was 15-25 years followed by 26-35 years (Table 1). Also, males were more commonly affected than females.

Table 1: Age And Sex Distribution In The Study

\begin{tabular}{|l|l|l|l|}
\hline Age group (years) & No. of cases & Male & Female \\
\hline$<15$ & 2 & 2 & 0 \\
\hline $15-25$ & 22 & 12 & 10 \\
\hline $26-35$ & 19 & 10 & 9 \\
\hline $36-45$ & 10 & 5 & 5 \\
\hline$>45$ & 2 & 1 & 1 \\
\hline
\end{tabular}

On histopathological and intraoperative examination, $5.45 \%$ had normal appendix, $54.54 \%$ had inflamed appendix, $21.81 \%$ had perforated appendix and $18.18 \%$ had gangrenous appendix respectively (Table 2).

Table 2: Histopathological Findings

Normal/Pathological appendix

\begin{tabular}{|l|l|l|}
\hline Normal appendix & No. of cases & Percentage \\
\hline Inflamed appendix & 3 & $5.45 \%$ \\
\hline
\end{tabular}


Volume - 10 | Issue - 12 | December - 2020 | PRINT ISSN No. 2249 - 555X | DOI : 10.36106/ijar

\begin{tabular}{|l|l|l|}
\hline \hline Perforated appendix & 12 & $21.81 \%$ \\
\hline Gangrenous appendix & 10 & $18.18 \%$ \\
\hline
\end{tabular}

Liver function tests were evaluated in these subjects, and it was found that in subjects with inflamed appendix, 5/30,3/30,2/30 had elevated levels of total bilirubin, AST, ALT respectively and none had elevated ALP. Similarly, in subjects with perforated/gangrenous appendix, $16 / 22,12 / 22,4 / 22$, and $2 / 22$ had elevated total bilirubin, AST, ALT and ALP levels respectively (Table 3 and 4 ).

Table 3: Liver Function Tests In Normal And Pathological Appendix

\begin{tabular}{|c|c|c|}
\hline & Normal appendix & Pathological appendix \\
\hline \multicolumn{3}{|c|}{\begin{tabular}{|l|} 
Total bilirubin \\
\end{tabular}} \\
\hline Elevated & 0 & 21 \\
\hline Normal & 3 & 31 \\
\hline \multicolumn{3}{|c|}{ AST/SGOT } \\
\hline Elevated & 0 & 15 \\
\hline Normal & 3 & 37 \\
\hline \multicolumn{3}{|c|}{ ALT/SGPT } \\
\hline Elevated & 0 & 6 \\
\hline Normal & 3 & 46 \\
\hline \multicolumn{3}{|l|}{ ALP } \\
\hline Elevated & 0 & 2 \\
\hline Normal & 3 & 50 \\
\hline
\end{tabular}

Table 4: Liver Function Tests In Pathological Appendix

\begin{tabular}{|l|l|l|}
\hline & $\begin{array}{l}\text { Inflammed } \\
\text { appendix }\end{array}$ & $\begin{array}{l}\text { Perforated/Gangrenous } \\
\text { appendix }\end{array}$ \\
\hline Total bilirubin & 5 & 16 \\
\hline Elevated & 5 & 6 \\
\hline Normal & 25 & 12 \\
\hline AST/SGOT & 3 & 10 \\
\hline Elevated & 27 & 4 \\
\hline Normal & 18 \\
\hline ALT/SGPT & 2 & \\
\hline Elevated & 28 & 2 \\
\hline Normal & 0 & 20 \\
\hline ALP & 0 & \\
\hline Elevated & 30 & \\
\hline Normal &
\end{tabular}

Table 5: Sensitivity, Specificity, PPV And NPV Of LFT Parameters In Perforated/gangrenous Appendicitis

\begin{tabular}{|l|l|l|l|l|}
\hline LFT parameters & Sensitivity & Specificity & PPV & NPV \\
\hline Total bilirubin & $72.72 \%$ & $84.84 \%$ & $76.19 \%$ & $82.35 \%$ \\
\hline AST/SGOT & $54.54 \%$ & $90.90 \%$ & $80 \%$ & $75 \%$ \\
\hline ALT/SGPT & $18.18 \%$ & $93.93 \%$ & $66.66 \%$ & $63.26 \%$ \\
\hline ALP & $9.09 \%$ & $100 \%$ & $100 \%$ & $62.26 \%$ \\
\hline
\end{tabular}

\section{DISCUSSION}

In the present study, out of 55 subjects, 30 were males and 25 were females. The study has shown male preponderance with $54.54 \%$ cases of acute appendicitis. Similar findings were discussed in one of the hospital based study done in Nepal. ${ }^{4}$ A majority of the study population was between 15 and 35 years. This is in concordance with the findings of Thangadurai Ramasamy Ramu et al. ${ }^{5}$

Normal appendix was seen in Histopathological findings of 3 cases $(5.45 \%)$ out of 55 cases in our study. Subedi et al. reported $1.4 \%$ (5 cases) of normal appendix in histopathological finding out of 345 cases. ${ }^{6}$

$54.54 \%$ of the cases in our study had inflamed appendix and $21.81 \%$ had perforated appendix and $18.18 \%$ had gangrenous appendix.

Liver function tests were evaluated in these subjects. We found that in subjects with inflamed appendix, 5/30 (16.6\%), 3/30 (10\%) and 2/30 $(6.6 \%)$ had elevated levels of total bilirubin, AST, ALT respectively and none had elevated ALP. Similarly, in subjects with perforated/gangrenous appendix, 16/22 (72.7\%), 12/22 (54.5\%), 4/22 $(18.2 \%)$ and $2 / 22(9.1 \%)$ had elevated total bilirubin, AST, ALT and ALP levels respectively. The LFT parameters are deranged in pathological appendix and more specifically the total bilirubin level is high for gangrenous and perforated appendix.

S Khan in his study reported that among 50 cases, 49 cases were of

acute appendicitis and its complications. Serum bilirubin was raised in $87.7 \%$ cases, Serum Alanine Amino Transferase (ALT) elevated in $26.54 \%$ of the case, Alkaline phosphatase (ALP) elevated in $48.98 \%$ of the cases and Amino Transferase (AST) was elevated in $38.77 \%$ of the cases.

Michael Sandet al in their study showed that patients with appendiceal perforation had a mean bilirubin level of $1.5 \mathrm{mg} / \mathrm{dL}$, which was significantly higher than those with a nonperforated appendicitis. ${ }^{8}$

Salvatore Giordano et al concluded from their study that, if symptoms and signs suggest perforated appendicitis, then serum bilirubin is one additional parameter in the primary evaluation. Hyperbilirubinemia alone is not a strong enough predictor, but might be more useful when integrated into a scoring system. ${ }^{9}$

Braja Mohan Mishra et al showed that $81.25 \%$ cases of complicated appendix and $21.42 \%$ of inflamed appendix have raised total bilirubin. $50 \%$ cases of complicated appendix have raised AST. ALT and ALP are raised in $15.62 \%$ and $18.75 \%$ cases of complicated appendix. ALT and ALP are not deranged in inflamed appendix. ${ }^{2}$

\section{SUMMARYAND CONCLUSION}

The Liver function test parameters are deranged in complicated acute appendicitis and the total bilirubin level is specifically increased in gangrenous and perforated appendix. The sensitivity, specificity, PPV and NPV for total bilirubin level are specifically high in gangrenous and perforated appendix. So LFT can help in the early diagnosis and management of the complications of acute appendicitis. This may also help in reducing the number of negative appendicectomies as specificity of the LFT parameters are quite high.

\section{REFERENCES}

1. Sisson, R. G., Ahlvin, R. C. \& Harlow, M. C. Superficial mucosal ulceration and the pathogenesis of acute appendicitis. Am. J. Surg. 122, 378-380 (1971).

2. Mishra, B. M., Nayak, M. K., Mishra, S., Sahu, P. \& Das, D. Role of liver function tes parameters in acute appendicitis and its complication: a prospective study. Int. Surg. J. 6, parameters in

3. Estrada, J. J. et al. Hyperbilirubinemia in appendicitis: A new predictor of perforation. in Journal of Gastrointestinal Surgery vol. 11 714-718 (2007).

4. Yadav, S. \& Chandra, K. Liver function test as a marker for acute appendicitis. Janaki Med. Coll.J. Med. Sci. 3, 31-36(2016).

5. Ramasamy Ramu, T., Chinnakkulam Kandhasamy, S., Andappan, A. \& Sankar T, B. A Prospective Study on the Diagnostic Value of Hyperbilirubinemia as a Predictive Factor for Appendicular Perforation in Acute Appendicitis. Cureus 10, (2018).

6. Subedi, N., Dangol, U., Adhikary, M., Pudasaini, S. \& Baral, R. Acute appendicitis: a 2year review of clinical presentation and histopathology. J. Pathol. Nepal 1, 104-107 (1970).

7. Khan, S. Effect of Acute Appendicitis on routine Liver Function Tests. J. Inst. Med. Nepal 30, 41-45(2008)

8. Sand, M. et al. Diagnostic value of hyperbilirubinemia as a predictive factor for appendiceal perforation in acute appendicitis. Am. J. Surg. 198, 193-198 (2009).

9. Giordano, S., Pääkkönen, M., Salminen, P. \& Grönroos, J. M. Elevated serum bilirubin in assessing the likelihood of perforation inacute appendicitis: A diagnostic metaanalysis. International Journal of Surgery vol. 11 795-800 (2013). 